\title{
Substance use risk profiles and associations with early substance use in adolescence
}

\author{
Monique Malmberg • Geertjan Overbeek • \\ Karin Monshouwer · Jeroen Lammers • \\ Wilma A. M. Vollebergh • Rutger C. M. E. Engels
}

Received: November 9, 2009/ Accepted: June 30, 2010/Published online: July 13, 2010

(c) The Author(s) 2010. This article is published with open access at Springerlink.com

\begin{abstract}
We examined whether anxiety sensitivity, hopelessness, sensation seeking, and impulsivity (i.e., revised version of the Substance Use Risk Profile Scale) would be related to the lifetime prevalence and age of onset of alcohol, tobacco, and cannabis use, and to polydrug use in early adolescence. Baseline data of a broader effectiveness study were used from 3,783 early adolescents aged 11-15 years. Structural equation models showed that hopelessness and sensation seeking were indicative of everused alcohol, tobacco or cannabis and for the use of more than one substance. Furthermore, individuals with higher levels of hopelessness had a higher chance of starting to use alcohol or cannabis at an earlier age, but highly anxiety sensitive individuals were less likely to start using alcohol use at a younger age. Conclusively, early adolescents who report higher levels of hopelessness and sensation seeking seem to be at higher risk for an early onset of substance use and poly substance use.
\end{abstract}

M. Malmberg $(\bowtie) \cdot$ R. C. M. E. Engels

Behavioural Science Institute, Radboud University Nijmegen,

P.O. Box 9104, 6500 HE Nijmegen, The Netherlands

e-mail: m.malmberg@pwo.ru.nl

G. Overbeek

Developmental Psychology, Utrecht University, Utrecht,

The Netherlands

K. Monshouwer · J. Lammers

Trimbos Institute (Netherlands Institute of Mental Health

and Addiction), Utrecht, The Netherlands

K. Monshouwer · W. A. M. Vollebergh

Department of Interdisciplinary Social Science,

Utrecht University, Utrecht, The Netherlands
Keywords Alcohol use - Tobacco use - Cannabis use · Personality $\cdot$ Early adolescence

\section{Introduction}

Dutch adolescents are one of the leaders in terms of drinking frequency and binge drinking in Europe and they usually start drinking in early adolescence (Hibell et al. 2009). Also, their use of tobacco and cannabis increases rapidly during this period (Monshouwer et al. 2008). This is disturbing in that early initiation of substance use has many detrimental consequences, like distortion of brain development (e.g., Tapert et al. 2002) and elevated risk for later dependence and misuse (e.g., Andersen et al. 2003). Further, early initiation increases the likelihood of poly substance use (Ellickson et al. 2003) that, in turn, leads to more damaging health effects (Feigelman et al. 1998). Thus, identifying risk profiles of early adolescent girls and boys is of crucial importance, because it may facilitate adequate prevention efforts targeted at youths who are at risk for an early onset of substance use or abuse (e.g., Conrod et al. 2008, 2010).

It is well known that personality is associated with substance use (e.g., Flory et al. 2002) and in general, personality dimensions involving neurotic tendencies or deficits in behavioral inhibition are found to best predict substance (mis)use (e.g., Barrett et al. 1998; Cloninger et al. 1991). Furthermore, personality dimensions concerning specific, rather than general personality dispositions are of most interest for substance related behaviors (Caspi et al. 1996; Comeau et al. 2001; Jackson and Sher 2003; Woicik et al. 2009). One instrument that specifically taps specific personality dimensions involving neurotic tendencies and inhibition deficits is the Substance Use Risk 
Profile Scale (SURPS; Woicik et al. 2009). This instrument measures four distinct and independent personality traits (i.e., anxiety sensitivity, hopelessness, sensation seeking, and impulsivity) that are hypothesized and actually appeared to be related to high and problematic substance use behaviors (Conrod et al. 1998; Jackson and Sher 2003; Pulkkinen and Pitkänen 1994; Shall et al. 1992; Sher et al. 2000; Stewart et al. 1995) and other risk behaviors (e.g., delinquency; Woicik et al. 2009).

The first trait (i.e., anxiety sensitivity) refers to the fear of symptoms of psychical arousal (e.g., feeling dizzy or faint; Reis et al. 1986) and the second (i.e., hopelessness) is identified as a risk factor for the development of depression (Joiner 2001). Both anxiety sensitivity and hopelessness relate to increased levels of drinking and problem drinking (Stewart et al. 1995; Conrod et al. 1998). The third trait (i.e., impulsivity) involves difficulties in the regulation (controlling) of behavioral responses (Spoont 1992) and is related to an increased risk for early alcohol and drug (mis)use (Pulkkinen and Pitkänen 1994). Finally, the fourth trait (i.e., sensation seeking) is characterized by the desire for intense and novel experiences (Zuckerman 1994) and sensation seekers have been found to drink more and to be more at risk for heavy alcohol use (Shall et al. 1992; Sher et al. 2000). The four SURPS' personality traits are based on extended personality measures (e.g., ASI; Peterson and Reiss 1992) and show stronger associations with these measures than with scales measuring broader dimensions of personality (e.g., NEO-FFI; Costa and McCrae 1992). Sensation seeking is, for instance, related to measures of openness and extraversion, but is more strongly related to scales measuring venturesomeness (Eysenck and Eysenck 1978; Woicik et al. 2009).

The SURPS personality traits show some overlap with traits of temperament (TCI; Cloninger 1998). Novelty seeking, for example, concerns the tendency to actively respond to new stimuli and thus reflects elements of impulsivity and sensation seeking. Further, the SURPS personality traits are relevant for more neuropsychological orientations. Different reinforcement processes are assumed to mediate the relationship between the SURPS personality traits and substance use in that the personality traits are susceptible to different types of reinforcement (e.g., Brunelle et al. 2004; Conrod et al. 1998). Individuals with high levels of anxiety sensitivity or hopelessness are more sensitive for the negative reinforcement processes of substance use (i.e., the ability of substances to relieve negative affective states). Individuals who score high on sensation seeking and impulsivity on the other hand are more sensitive for the positive reinforcement processes of substance use (i.e., the positive hedonic effects of a substance).
According to Carver et al. (2009) these processes are even more apparent in case of low serotonergic function. It is argued that individual differences in serotonergic function are important for personality dispositions in that individuals with low serotonergic function are especially susceptible for (affective) cues of the moment (Spoont 1992), like reinforcement processes. In accordance, low serotonergic function is related to personality dispositions as sensation seeking, impulsivity, and depression (Carver et al. 2009). Considering the possible contribution of the SURPS to many different fields (e.g., neuropsychology), the fact that a more clinical orientation (i.e., the use of more clinical instruments like the TCI) seems less obvious for early adolescents who are in the beginning stage of substance use, and bearing in mind that specific rather than general personality traits are most interesting, the SURPS is a potentially important measurement for examining the role of personality on substance use behaviors.

Recall that the SURPS-based personality profiles are useful in identifying individuals who are at risk for alcohol use and alcohol-related problems in already using samples. However, to our knowledge no previous study examined whether these personality profiles are indicative of an early onset of alcohol, tobacco, cannabis, and poly substance use. This is unfortunate, because on the one hand early initiation is one of the strongest identified risk factors for alcohol (De Wit et al. 2000), tobacco (Breslau et al. 1993), and cannabis problems (Chen et al. 2005) in later life. Further, poly substance use in adolescence is a significant predictor of poly substance use in adulthood (Galaif and Newcomb 1999). On the other hand, the developmental role of personality dispositions is important. The lower order personality dispositions might be overruled by higher order systems (i.e., rational or cognitive), but only if and once the capacity for behavioral control develops (i.e., through maturation of the pre-frontal cortex; Carver et al. 2009). Thus, one might argue that especially early adolescents are vulnerable for these lower order personality predispositions. To conclude, focusing on early onset of substance use in early adolescence, and identifying the specific personality profiles related to these risk behaviors, might help us to identify youngsters at an early age who are at risk for developing future substance misuse patterns.

The present study examines a SURPS-based, four-factor personality model in relation to early onset substance use and poly substance use. A total of 3,783 adolescents in the ages of 11-15 participated in the first wave of the ongoing Healthy School and Drugs (HSD) effectiveness study in which they filled out a digital questionnaire. Participants 
answered questions about alcohol, tobacco, and cannabis use and their personality traits. Based on previous research on personality, we expected to find strongest associations with substance use for sensation seeking. Specifically, we hypothesize sensation seekers to have an increased risk for an early initiation of alcohol, tobacco, and cannabis use. Hence, we expected to find that anxiety sensitive adolescents have an increased risk for an early onset of alcohol use, adolescents reporting higher levels of hopelessness to have an increased risk for an early onset of alcohol and tobacco use, and impulsive adolescents to have an increased risk for an early onset of alcohol and cannabis use. Following these expectations we also expected to find associations between the SURPS personality profiles and poly substance use. However, considering the lack of knowledge so far in adolescence, no concrete expectations were formulated on poly substance use.

\section{Method}

Sample and procedure

The cross-sectional data for this study were collected as part of a broader effectiveness study on a national school prevention program "The Healthy school and drugs." A total of 23 schools were included from seven regions in The Netherlands. We visited participating schools and during these visits we provided further information about the research project. In collaboration with the schools' headmasters, we informed the students' parents about the goals of the study by a letter in which parents were also explained they could refuse participation of their child in the study. Approval for the design and data collection procedures was obtained from the ethic committee of the Radboud University Nijmegen. All data were collected between January and March 2009. All first grade students independently filled out a digital questionnaire during school hours in the presence of a teacher and a research assistant. The questionnaires were counterbalanced on alcohol, tobacco, and cannabis, thus six different versions were administrated.

In total, 3,783 first-grade students took part in the study of whom 231 (6.1\%) were absent (i.e., illness) during datacollection and three participants were declined participation by their parents. The total sample included 1,856 boys $(49.1 \%)$ and $31.5 \%(n=1,192)$ of all participants pursued lower secondary vocational education, $46.6 \%(n=1,764)$ pursued pre-university education, and $21.9 \%(n=827)$ of the students pursued a mixed educational program. Of the participants who completed the questionnaire 3,375 participants $(96.2 \%)$ were of Dutch ethnic origin. Students ranged in age from 11 to 15 years $(M=13.01, \mathrm{SD}=.49)$. For the question on lifetime prevalence of alcohol use, $2,103(59.9 \%)$ reported to have at least once used alcohol in the past. With regard to smoking, 768 (22.1\%) participants had ever smoked, and with regard to cannabis 75 (2.1\%) participants reported to have at least once used cannabis. Finally, $670(19.6 \%)$ stated that they already had tried more than one substance.

\section{Measures}

\section{Personality profiles}

The Substance Use Risk Profile Scale (SURPS; Woicik et al. 2009) distinguishes four personality dimensions, namely anxiety sensitivity, hopelessness, sensation seeking, and impulsivity. Each dimension was assessed using five to seven items that could be answered on a 4-point scale, ranging from $1=$ 'strongly agree' to $4=$ 'strongly disagree.' Anxiety sensitivity refers to the fear for physical arousal and an example item is: 'It's frightening to feel dizzy or faint.' Hopelessness concerns negative thinking which might lead to depression proneness and 'I feel that I'm a failure' is an example item. Sensation seeking is characterized by wanting to try out new things and an example of such an item is 'I like doing things that frighten me a little.' Finally impulsivity refers to having difficulties in controlling behavioral responses, and 'I usually act without stopping to think' is an example item. Factor structure, internal consistency and test-retest reliability, as well as construct, convergent, and discriminant validity of this instrument were shown to be adequate in studies among college students and adult samples (e.g., Krank et al. submitted). Because the instrument was translated in Dutch and used for the first time the factor structure was examined using Exploratory Factor Analysis (EFA) on a randomly selected sample that consisted of the first half of the original sample using Mplus (Muthén and Muthén 1998-2007). The Weighted Least Square parameter estimator with Mean- and Variance adjusted chi-square test statistic (WLSMV) was used because the metric of the items is more ordered categorical than interval level. The sample was randomly divided into two subsamples. Two items were removed. The first item (i.e., I feel that I'm a failure) had substantial loadings (.38 and .42 , respectively) on the factors anxiety sensitivity and hopelessness. The second item (i.e., I feel I have to be manipulative to get what I want) showed an almost zero loading on the factor impulsiveness. A Confirmatory Factor Analysis (CFA) was performed on the remaining 21 SURPS items on the other half of the sample and confirmed the four-factor structure of the SURPS. The final model had a satisfactory fit to the data $\left(\chi^{2}(54)=611.315, P<.001\right.$, RMSEA $=.055$, 
$\mathrm{CFI}=.943$ ). Cronbach's alphas were .69 for anxiety sensitivity (factor loadings between .42 and .72), .85 for hopelessness (loadings between .72 and .96 ), .68 for sensation seeking (loadings between .38 and .72 ), and .67 for impulsivity (loadings between .48 and .72). These reliability estimates converge with those from previous research (e.g., Jaffee and D'Zurilla 2009) and are satisfactory for short scales (Loewenthal 1996).

\section{Substance use}

We assessed adolescents' alcohol use in terms of lifetime prevalence, or whether participants had ever consumed alcohol in their life. Lifetime prevalence was measured by asking: "Have you ever drunk alcohol?" Participants could answer this question with yes $(=1)$ or no $(=0)$. To determine the age of onset of participants' alcohol use we asked how old they were when they had first drunk alcohol (Kuntsche et al. 2009).

Lifetime prevalence of tobacco use was measured by a single item on a 9-point scale ranging from $1=$ 'I never smoked, not even a puff' to $9=$ 'I smoke at least once a day' (Kremers et al. 2001). To tap lifetime prevalence of smoking, adolescents who responded in the categories 2-9 were categorized as tried smoking before $(=1)$, and the adolescents who responded in category 1 were categorized as never tried smoking (=0) following Kremers (2002). In order to assess age of onset, participants who had ever smoked were asked how old they were when they smoked their first puff.

We assessed the lifetime prevalence of cannabis use through a single item, namely: "Have you ever used cannabis?" (Monshouwer et al. 2005). Participants could answer with yes $(=1)$ or no $(=0)$. Subsequently, participants who ever used cannabis were asked how old they were when they first used cannabis.

Finally, poly substance use was operationalized by the use of more than one substance, regardless of the combination or amount of substances used. A new variable was created in which all adolescents who used more than one substance were categorized as poly substance users $(=1)$ and all other adolescents as non-poly substance users $(=0)$.

\section{Strategy of analyses}

First, descriptive analyses and Pearson correlations of age of onset of alcohol, tobacco, and cannabis use and the personality profiles (i.e., anxiety sensitivity, hopelessness, sensation seeking, and impulsivity) were calculated between model variables. Second, to investigate whether participants' sex and educational level should be specified as covariates in the model, a MANOVA was conducted to compare responses on the SURPS personality profiles between males and females and between different educational levels. Another MANOVA was carried out to investigate sex and educational differences on substance use. Also, separate ANOVA's were conducted to examine sex and educational level differences on age of onset of alcohol, tobacco, and cannabis use. The effect sizes (i.e., partial eta squared) are reported for the analyses of variance. With respect to the effect size, values around .02 are considered small effects, values around .15 medium effects, and values around .35 large effects (Cohen 1992). Post-hoc tests with Bonferroni corrections were carried out to investigate the significant differences in educational level on the outcome variables.

Next, to investigate the relationships between personality profiles and lifetime prevalence of alcohol, tobacco, and cannabis use, we specified and tested a first model (see Fig. 1) with structural equation modeling (SEM) in Mplus (Muthén and Muthén 1998-2007). In this model, lifetime prevalences of alcohol, tobacco, and cannabis were included as observed variables and personality profiles were added as latent constructs, with separate scale items as indicators. Sex and educational level were specified as covariates in the model. We used the weighted least square method (WLSMV) to estimate parameters in the model. The Chi-square and the p-value, the Comparative Fit Index (CFI: Bentler 1989), and the

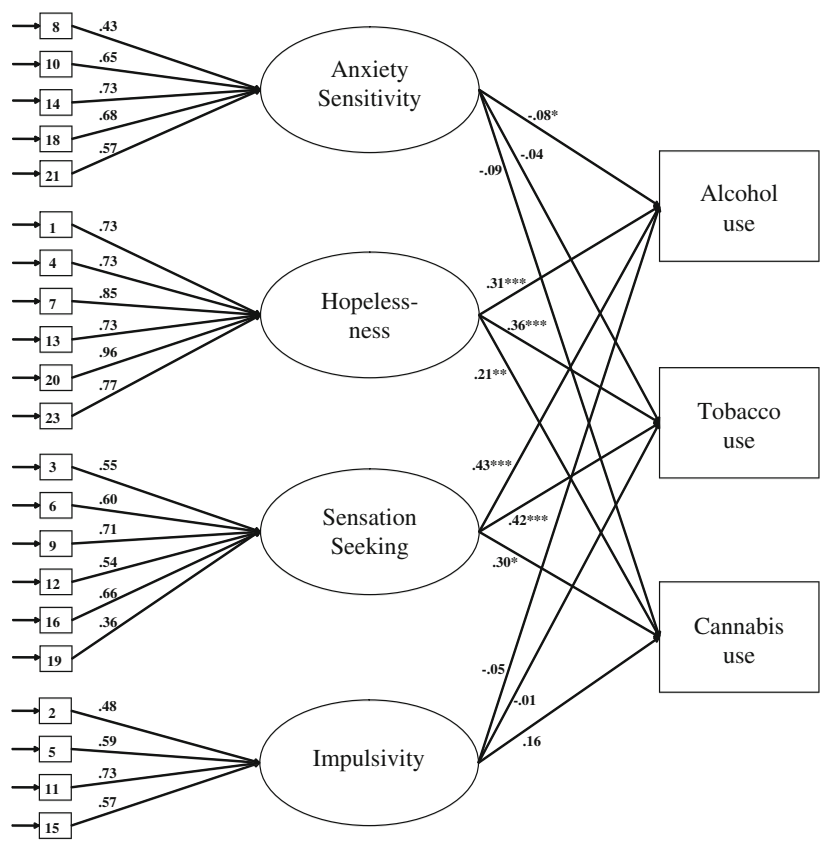

Fig. 1 Standardized estimates of associations between SURPS personality profiles and lifetime prevalence of substance use $(n=3,783)$. $* P<.05, * * P<.01, * * * P<.001$ 
Root Mean Square Error of Approximation (RMSEA: Steiger 1990) were used to assess the goodness of fit of the model. With respect to the CFI, values above .90 indicate an acceptable fit and values above .95 signify an excellent fit to the data. Concerning the RMSEA, values below .08 point to an acceptable fit and values below .05 indicate a good fit of the model to the data ( $\mathrm{Hu}$ and Bentler 1999). The explained variance was used as a measure of effect size. Values around $2 \%$ are considered small, values around $15 \%$ medium, and values around $35 \%$ are considered large effects (Cohen 1992). The data have a multilevel structure (i.e., data of individual students are nested within classes), which means that apart from differences between individuals, average substance use levels across classes may vary as well. In particular, participants within certain classes may be more similar to each other due to specific influence and selection processes (Kuntsche et al. 2008); classmates in our target group might influence each other in such a way that their substance using behaviors become more similar. This means that individual respondents are not independent within classes. As a consequence the standard errors of the parameter estimates are biased leading to incorrect decisions about the significance of parameter estimates. The COMPLEX procedure in Mplus is used to correct for dependency of the data, which results in unbiased standard errors (cf Kuntsche and Jordan 2006).

To investigate the relationship between personality profiles and age of onset of alcohol, tobacco, and cannabis use only substance users were included in the subsequent analyses (e.g., only those who already drank alcohol were included in the analysis to see whether personality profiles were related to the age of onset of alcohol use). The personality profiles were again included in the model as latent constructs and the age of onset as an observed variable. Identical statistical procedures were used as in the former model. Finally, to investigate the relationship between personality profiles and poly substance use two variables were created in the dataset, one for mono use and one for poly use. All participants that only used one substance were assigned a score ' 1 ' and all others were assigned ' 0 ' in the mono variable. For poly substance use, all participants who reported having used two or three substances were assigned ' 1 ' and all others were assigned ' 0 '. Based on this information, we estimated the two models with the same procedures as the other models in Mplus.

\section{Results}

Descriptive analyses

Table 1 presents the means and standard deviations of the SURPS' personality profiles and age of onset examined in the present study, separately for educational level and sex. For Pearson correlations of the model variables we refer to "Appendix". A MANOVA was conducted to examine whether personality profiles would significantly differ across sex and educational level. Main effects of sex $[F(4$, $\left.3,431)=86.40, P<.001, \eta_{p}^{2}=.092\right]$ and education $[F(8$, $\left.6,862)=15.92, \quad P<.001, \eta_{p}^{2}=.018\right]$ emerged in the MANOVA on different personality profiles. Univariate tests showed sex effects for anxiety sensitivity $[F(1$, $\left.3,434)=110.79, P<.001, \eta_{p}^{2}=.031\right]$, hopelessness $[F(1$, $\left.3,434)=5.50, P=.02, \eta_{p}^{2}=.002\right]$, and sensation seeking $\left[F(1,3,434)=212.69, P<.001, \eta_{p}^{2}=.058\right]$. Specifically, we found that girls reported higher scores on anxiety sensitivity and hopelessness than boys, and boys reported higher levels of sensation seeking than girls. Associations were also found between education and hopelessness

Table 1 Means and standard deviations for personality profiles and age of onset

\begin{tabular}{|c|c|c|c|c|c|c|}
\hline & \multicolumn{2}{|l|}{ Gender } & \multicolumn{3}{|c|}{ Educational level } & \multirow[t]{2}{*}{ Total } \\
\hline & Female & Male & Lower & Mixed & Higher & \\
\hline \multicolumn{7}{|l|}{ Age of onset } \\
\hline Alcohol & $10.40(2.19)^{*}$ & $9.67(2.50)^{*}$ & $10.41(2.44)^{\mathrm{ab}}$ & $9.93(2.40)^{\mathrm{a}}$ & $9.77(2.30)^{\mathrm{b}}$ & $10.01(2.38)$ \\
\hline Tobacco & $11.26(1.67)^{*}$ & $10.91(1.99)^{*}$ & $11.31(1.79)^{\mathrm{ab}}$ & $10.90(2.01)^{\mathrm{a}}$ & $10.81(1.81)^{\mathrm{b}}$ & $11.07(1.86)$ \\
\hline Cannabis & $12.45(.74)$ & $11.90(1.53)$ & $12.28(1.08)$ & $11.75(1.24)$ & $11.75(2.18)$ & $12.07(1.36)$ \\
\hline \multicolumn{7}{|l|}{ Personality profiles } \\
\hline Anxiety sensitivity & $2.38(.62)^{*}$ & $2.13(.67)^{*}$ & $2.30(.72)^{\mathrm{a}}$ & $2.27(.66)$ & $2.23(.62)^{\mathrm{a}}$ & $2.26(.66)$ \\
\hline Hopelessness & $1.55(.53)^{*}$ & $1.50(.56)^{*}$ & $1.64(.63)^{\mathrm{ab}}$ & $1.51(.51)^{\mathrm{a}}$ & $1.46(.49)^{\mathrm{b}}$ & $1.52(.55)$ \\
\hline Sensation seeking & $2.38(.66)^{*}$ & $2.72(.66)^{*}$ & $2.49(.70)^{\mathrm{ab}}$ & $2.60(.69)^{\mathrm{a}}$ & $2.56(.67)^{\mathrm{b}}$ & $2.55(.68)$ \\
\hline Impulsivity & $2.18(.59)$ & $2.23(.64)$ & $2.29(.66)^{\mathrm{a}}$ & $2.24(.61)^{\mathrm{b}}$ & $2.14(.58)^{\mathrm{ab}}$ & $2.21(.62)$ \\
\hline
\end{tabular}

Means with the same superscripts are significantly different from each other. All at $P<.05$ with Bonferroni corrections for educational level 
$\left[F(2,3,434)=36.40, P<.001, \eta_{p}^{2}=.021\right]$, sensation seeking $\left[F(2,3,434)=9.73, P<.001, \eta_{p}^{2}=.006\right]$, and impulsivity $\left[F(2,3,434)=21.88, P<.001, \eta_{p}^{2}=.013\right]$. Students of higher education reported higher scores on impulsivity and hopelessness compared to students of lower education. The pattern for sensation seeking was somewhat different. Students of mixed education reported higher scores than students in both lower and higher educational levels, but students of higher education scored higher than students of lower education.

Another MANOVA was conducted to look at possible differences for sex and educational level on substance use. We found main effects for both sex $[F(4,3,411)=11.04$, $\left.P<.001, \eta_{p}^{2}=.013\right]$ and education $[F(8,6,822)=23.80$, $\left.P<.001, \eta_{p}^{2}=.027\right]$ on substance use. Univariate tests showed sex effects for alcohol $[F(1,3,414)=23.98, P<$ $\left..001, \quad \eta_{p}^{2}=.007\right]$, tobacco $[F(1,3,414)=17.72, \quad P<$ $\left..001, \eta_{p}^{2}=.005\right]$, cannabis $[F(1,3,414)=17.51, P<.001$, $\left.\eta_{p}^{2}=.005\right]$, and poly substance use $[F(1,3,414)=17.75$, $\left.P<.001, \eta_{p}^{2}=.005\right]$. Particularly, we found that more boys already used the different substances compared to girls and more boys were poly substance users in contrast to girls. Univariate tests also showed education effects on alcohol $\left[F(2,3,414)=3.32, P=.04, \quad \eta_{p}^{2}=.002\right]$, tobacco $\left[F(2,3,414)=88.89, P<.001, \eta_{p}^{2}=.049\right]$, cannabis $\left[F(2,3,414)=17.96, P<.001, \eta_{p}^{2}=.010\right]$ and poly substance use $\left[F(2,3,414)=70.68, P<.001, \eta_{p}^{2}=.040\right]$. More students of lower education reported having used alcohol, tobacco, or cannabis compared to students from higher education. Also, students of lower education were more likely to use more than one substance compared with students from higher education.

We conducted a set of three ANOVA's to test sex and education differences for age of onset of alcohol use, tobacco use, and cannabis use. Main effects of sex $[F(1,2,038)=$ 51.07, $\left.P<.001, \eta_{p}^{2}=.024\right]$ and education $[F(2,2,038)=$ $\left.14.05, P<.001, \eta_{p}^{2}=.014\right]$ were found for the age of onset of alcohol. With regard to tobacco use we found main effects of $\operatorname{sex}\left[F(1,745)=5.65, P=.02, \eta_{p}^{2}=.008\right]$ and education $\left[F(2,745)=5.20, P<.01, \eta_{p}^{2}=.014\right]$. Finally, the last ANOVA in which age of onset of cannabis use was the dependent variable, showed no main effects for sex and education. In sum, the results indicated that boys and students from higher education start drinking and smoking earlier compared to girls and students from lower education. Overall, although the effects of sex and educational level on substance use and personality were small, they were still significant and were therefore specified as covariates in the subsequent analyses.
Personality profiles and lifetime prevalence

The model as depicted in Fig. 1 showed a good fit to the data $\left[\chi^{2}(d f=68, n=3,783)=725.791, P<.001\right.$, RMSEA $=.051, \mathrm{CFI}=.929]$. As can be seen in Fig. 1, standardized estimates for the associations between personality profiles and lifetime prevalences revealed significant associations between anxiety sensitivity $(\beta=-.08$, $P=.024)$, hopelessness $(\beta=.31, P<.001)$, and sensation seeking $(\beta=.43, P<.001)$ with the lifetime prevalence of alcohol use. These results indicate that youngsters with lower levels of anxiety sensitivity and higher levels of hopelessness and sensation seeking were more likely to have ever consumed alcohol. Further, we found significant associations between hopelessness $(\beta=.36$, $P<.001)$ and sensation seeking $(\beta=.42, P<.001)$ with the lifetime prevalence of tobacco use. Adolescents who were high on hopelessness and sensation seeking were more likely to have ever smoked than adolescents who were low on these two profiles. Finally, the analysis showed significant linkages between hopelessness $(\beta=.21, P=.007)$, sensation seeking $(\beta=.30, P=$ .023 ) and lifetime prevalence of cannabis use. This means that youngsters who had higher levels of hopelessness and sensation seeking had a higher chance of having ever used cannabis at this age than youngsters who had lower scores on these profiles. The models showed medium to large effect sizes for the relationships between the four personality profiles and substance use; they explained $19.1 \%$ of the variance in lifetime prevalence of alcohol use, $31.3 \%$ of the variance in tobacco use, and $28.8 \%$ of the variance in cannabis use.

Personality profiles and age of onset

The model that specified the relationship between personality profiles and the age of onset of alcohol use showed an adequate fit to the data $\left[\chi^{2}(d f=62, n=2,103)=\right.$ 416.739, $P<.001$, RMSEA $=.052$, CFI $=.943]$. Controlling for participants' sex and education, we found significant associations between hopelessness and age of onset of alcohol use (Table 2). This result showed that students start to drink at a younger age when they have higher levels of hopelessness. The model that assessed the relationship between personality profiles and age of onset of tobacco use also showed an adequate fit to the data $\left[\chi^{2}(d f=58\right.$, $n=768)=228.326, P<.001$, RMSEA $=.062, \mathrm{CFI}=$ .928]. Table 2 shows the standardized estimates of this model; we did not find any significant associations between the personality profiles and the age of onset of tobacco use. 
Table 2 Standardized estimates and standard errors for tested models

\begin{tabular}{|c|c|c|c|c|c|c|c|c|c|c|}
\hline & \multicolumn{6}{|c|}{ Age of onset } & \multicolumn{4}{|c|}{ Substance use } \\
\hline & \multicolumn{2}{|l|}{ Alcohol } & \multicolumn{2}{|c|}{ Tobacco } & \multicolumn{2}{|c|}{ Cannabis } & \multicolumn{2}{|l|}{ Mono } & \multicolumn{2}{|l|}{ Poly } \\
\hline & $\beta$ & SE & $\beta$ & SE & $\beta$ & SE & $\beta$ & SE & $\beta$ & SE \\
\hline Anxiety & .01 & .03 & .03 & .06 & .01 & .11 & -.04 & .04 & -.07 & .04 \\
\hline Hopelessness & $-.10 * *$ & .04 & -.04 & .05 & $-.37 * *$ & .17 & $.22 * * *$ & .04 & $.37 * * *$ & .04 \\
\hline Sensation & -.06 & .05 & .11 & .09 & -.22 & .10 & $.34 * * *$ & .06 & $.43 * * *$ & .06 \\
\hline Impulsivity & -.05 & .05 & -.09 & .08 & -.02 & .10 & -.05 & .07 & .01 & .07 \\
\hline
\end{tabular}

** $P<.01, * * * P<.001$

We could not adequately test the relationship between personality profiles and age of onset of cannabis use considering the small sample size of cannabis users $(n=75)$. As an alternative (to reduce the number of parameters to be estimated) we applied regression analysis in Mplus with sex and education as control variables and the four manifest personality profiles as predictors of age of onset of cannabis use. We found a significant relationship between hopelessness and age of onset of cannabis use ( $\beta=-.37, P=.001$ ) indicating that an increase of hopelessness is associated with a decrease of age of onset of cannabis use. The models showed small effect sizes for the association between the four personality profilescontrolling for sex and educational level-and the age of onset of alcohol $\left(R^{2}=5 \%\right)$ and tobacco $\left(R^{2}=3.3 \%\right)$ use, and a medium effect size for the relationship between personality profiles, sex and educational level on the one hand and the age of onset of cannabis use on the other $\left(R^{2}=17.7 \%\right)$.

Personality profiles and poly substance use

The mono substance use model showed a good fit to the data $\left[\chi^{2}(d f=62, n=3,783)=656.514, P<.001\right.$, RMSEA $=.050, \quad$ CFI $=.937]$. Significant associations were found between hopelessness and sensation seeking with mono substance use (Table 2). Thus, students that experienced more feelings of hopelessness or students who were higher on sensation seeking were also more likely to use one specific substance (i.e., either alcohol, tobacco, or cannabis). The model examining poly substance use showed a good fit to the data $\left[\chi^{2}(d f=62, n=3,783)=\right.$ 693.229, $P<.001$, RMSEA $=.052, \mathrm{CFI}=.933]$. The results in Table 2 display significant associations between hopelessness, sensation seeking, and poly substance use. Thus, more feelings of hopelessness and being a sensation seeker were related to the use of more than one substance. The model on mono-substance use showed a medium effect size $\left(R^{2}=11.4 \%\right)$ for the four personality profiles and the model on poly substance use a large effect size $\left(R^{2}=31.8 \%\right)$.

\section{Discussion}

The results clearly demonstrated that, overall, three out of the four SURPS' personality profiles are associated with early adolescents' substance use behavior. Notably, the different models revealed that-in this sample of early adolescents, of whom many are in the starting phase of experimentation with substance use-especially hopelessness and sensation seeking are strongly associated with a higher chance of ever-used alcohol, tobacco, and cannabis at an early age and with poly substance use. Individuals with higher levels of hopelessness have also a higher chance of starting to use alcohol or cannabis at an earlier age. Highly anxiety sensitive individuals on the other hand are less likely to start using alcohol use at a younger age.

Personality profiles and lifetime prevalence

Previous studies investigating the role of the SURPS personality profiles on alcohol use mainly focused on more advanced levels of drinking (e.g., Cooper et al. 1995). Our present results extend this knowledge by demonstrating that the revised SURPS personality profiles are not only indicative of already established maladaptive drinking patterns in adolescents and adults (e.g., Sher et al. 2000), but are also associated with alcohol use in young adolescents. Specifically, the SURPS personality profiles are associated with early adolescents' alcohol use to a moderately strong degree. For this particular age group, we found that especially hopelessness and sensation seeking are indicative of having ever used alcohol in early adolescence. The results with regard to sensation seeking are not unexpected given the novelty seeking nature of sensation seekers and that experimenting with 
different substances can be seen as such novel experiences. Although it was not clear what the role of hopelessness would be in our age group, we did find it surprising that hopelessness seems this important in our age group, since this trait was primarily found to be predictive of a progression into substance misuse before (e.g., Jackson and Sher 2003). One possible explanation is that hopelessness leads adolescents to initiate substance use as a means to cope with negative thoughts. Therefore, we examined if higher scores on different coping strategies (e.g., drinking alcohol makes me relaxed) were related to higher levels of hopelessness. However, we could not substantiate this explanation based on these additional analyses of our data. More information on these analyses can be obtained from the first author.

Another explanation is that early childhood problems (e.g., family violence, unorganized family environments, antisocial behavior) can lead to both negative affect (e.g., Reinherz et al. 2003) and an early onset of substance use (e.g., Dishion et al. 1999). The existing relationship between hopelessness and the lifetime prevalences might then be based on a third variable explanation, indicating that early childhood adversity can affect the development of personality profiles, and subsequent engagement in problem behaviors (Akse et al. 2004; Hale et al. 2008). Since hopelessness is associated with self-harm and suicide behavior (O'Connor et al. 2008), there might also be a link between hopelessness and more 'nihilistic' behaviors. Further research is necessary to disentangle the potential pathways in which hopelessness is related to early substance use behaviors. Contradictive to our expectations we found a negative association between anxiety sensitivity and alcohol use. This can be explained by the preventive effect that the fear for physical arousal might have. When highly anxiety sensitive individuals have no prior experience with alcohol they also do not know if drinking alcohol leads to unusual body sensations, which might keep them from drinking. Also, it could be that highly anxiety sensitive individuals are more anxious in general, and are for instance afraid of loosing control when drinking.

Our findings also indicate a clear linkage between two personality profiles (i.e., hopelessness and sensation seeking) and ever-used tobacco in early adolescence. The few studies that investigated the role of personality (i.e., Big Five) on lifetime smoking in adolescence (Harakeh et al. 2006; Otten et al. 2008) found extraversion and openness to be risk factors for lifetime smoking and conscientiousness, agreeableness, and emotional stability to be protective factors. Our results are in line with these latter findings, considering that extraversion and openness are more strongly related to sensation seeking and hopelessness is at the opposite end of emotional stability. Finally, our results show that sensation seeking is associated with an early onset of cannabis use. This is in line with previous results showing that sensation seeking predicts reckless behavior, like cannabis use (Arnett 1994). It is thought that sensation seekers use substances for the euphoric/intoxicating effects (Comeau et al. 2001), so it might be that especially sensation seekers attribute such characteristics to different substances (e.g., cannabis) and are therefore more likely to initiate use of a certain substance. We also found an association between hopelessness and having ever used cannabis in early adolescence. It is again not quite clear yet how to interpret this finding in our age group. Previous results in older adolescents suggest that hopelessness also predicts reckless behavior, but particularly with regard to the use of cocaine and other illegal drugs, not cannabis (Woicik et al. 2009). Also, for this finding it might be that early childhood problems directly affected both hopelessness and the use of cannabis. Overall, the fact that the SURPS personality profiles are related to early adolescents' tobacco and cannabis use to a moderately strong degree indicate that these profiles are important in explaining individual differences in early adolescent substance use behaviors.

\section{Personality profiles and age of onset}

We only found support for the role of hopelessness on the age of onset of alcohol and cannabis use. We believe that these findings might also be explained by the third variable (i.e., early childhood problems) explanation. Besides the findings considering hopelessness we hardly found any support for the relationship between the personality profiles and the age of onset of the different substances. It could be that this outcome is due to the retrospective character of these questions or to the restriction of range. Adolescents were asked the age when they had their first experience with a specific substance. In The Netherlands, most adolescents start experimenting first with alcohol, followed by tobacco and cannabis (Monshouwer et al. 2008). So, especially with respect to alcohol and tobacco use, the recollection time between the first experience and the moment of questioning is longer, and might thus be less adequate (Bailey et al. 1992; Engels et al. 1997). Simultaneously, this trend causes differences in the diversity of answers. Since youngsters start using cannabis at a later age, less variation is visible in the ages of onset compared to the start of using alcohol or tobacco. These effects could explain the lack of findings on age of onset of cannabis use and might explain the small effects found for the associations between the SURPS personality profiles and age of onset. 
Personality profiles and poly substance use

In the present study, we found that the SURPS personality profiles are strongly related to early adolescents' poly substance use. Specifically, we found that hopelessness and sensation seeking are indicative of poly substance use and these results are mostly in line with earlier findings. Previous studies suggested that poly substance users have particularly high levels of impulsivity and sensation seeking (e.g., Galizio and Stein 1983; Lacey and Evans 1986). Also, there is evidence suggesting that poly substance users are low on agreeableness and conscientiousness and high on neuroticism (McCormick et al. 1998). Many of these studies examined the relationship between personality and poly substance use in a clinical (i.e., substance dependent) sample and as far as we know little is known about the early onset of poly substance use in young adolescents. In contrast to these findings, although we found a strong link for hopelessness and sensation seeking with poly substance use, we did not find a relationship between impulsivity and poly substance use. In the present study we defined poly substance use by the use of more than one substance, comprising alcohol, tobacco, and/or cannabis use. Other studies among older or clinical samples usually operationalized poly substance use by the use of multiple (hard) drugs, like cocaine, xtc, and opiates (e.g., Smit et al. 2002). So, it might be that impulsivity only has sufficient dicriminant power in poly substance use, when the use of certain substances is deviant enough.

Strengths, limitations, and implications for future research

A major strength of our study is the large representative non-clinical sample of our study. In addition, instead of exclusively examining adolescents' alcohol use we also focused on tobacco and cannabis use. The large sample allowed us to perform sophisticated SEM analyses in which we controlled for the multilevel structure of the data. Finally, a strength of the study is that our measurements were well-validated and had all good psychometric properties.

Some limitations were present in the current study as well. First of all, a cross-sectional design was used-thus, no causal explanations can be based on these associations. Roberts et al. (2006) found in their meta-analysis that the mean level of personality traits changed across the life course, especially during adolescence. In general, it is found that one's personality type is only moderately stable in childhood (e.g., Hart et al. 2003) and adolescence (e.g., Akse et al. 2007). So, do personality profiles precede substance use behaviors or do experiences with substance use modify personality profiles? We investigated the role of personality in substance use in a group of early adolescents that is in their initiation phase of alcohol and tobacco use and has hardly any experience with cannabis. One might thus question if the potential changes in personality due to substance (ab)use are already noticeable in these early adolescents. It seems more likely that these changes will become apparent in a later stage, when adolescents have more experience with substance use or when more time has gone by after the actual initiation of substance use. It seems plausible to assume that, in a group of early adolescents who are in their starting phase of substance use, personality precedes substance use behaviors. However, this assumption should be interpreted carefully, since longitudinal research is required to shed more light on this topic.

Secondly, the fit of the models expressed in RMSEA varied between .050 and .062 , the CFI varied between .928 and .943. This means that the fit of the models were acceptable but not excellent. There is ample literature about fit indices and cut-off scores. In our view, an important reason for the absence of excellent fit is related to the measurement part of the models (the factor model). In the factor model a simple structure is required with cross-loadings constrained to be zero. In exploratory factor models cross loadings are admitted resulting in better fitting models. We applied a newly developed exploratory structural equation model (ESEM) on the models in this article. In these models the measurement part of the structural model is estimated by the exploratory factor model (Asparouhov and Muthén 2009). In fact, the confirmatory factor model in the structural model was replaced by an exploratory factor model. The fit of all models were improved with CFI-values $>.95$ and RMSEA-values $<.05$. Because the structural parameters did not change substantially we preferred to use the classical SEM model with the confirmatory factor model as measurement model.

Thirdly, our use of self-reports might have lead to measurement errors. Two perspectives can explain possible measurement errors in self-reports on substance use, namely a situational and a cognitive perspective (Brener et al. 2003). The situational perspective concerns the influence of the social environment, which might lead adolescents to give socially desirable answers. To avoid social desirability and optimize measurement validity we guaranteed full confidentiality (anonymity) to our participants (e.g., Dolcini et al. 1996). The cognitive perspective concerns the cognitive or internal processes that might influence the self-reports. They might over or underestimate their substance use behaviors in that they can not 
exactly recall what they have been using in a certain period (e.g., Engels et al. 1997). In our study we asked participants if they ever tried a specific substance, which is arguably different from asking them how much they have used in a certain period. One might expect participants to reliably recall ever using alcohol, tobacco, or cannabis before. With respect to the questions on age of onset the cognitive aspect seems more relevant, thus one might argue that more measurement errors occurred in these self-reports. However, the time between the age of first drink and assessment seems to matter. The longer the time interval the more severe recall bias one might expect (e.g., Engels et al. 1997; Parra et al. 2003). In our study, we investigated the age of onset in a group of early adolescents with an average age of 13 and assessing the reported age of onset close to the actual age will optimize the reliability of the self-reports (Kuntsche et al. 2009).

Fourthly, we only focused on the relationship between the SURPS personality profiles and substance use behaviors. It would be interesting to investigate if the SURPS personality profiles are also indicative of other risk type behaviors. Finally, in our design we used a variable-centered approach utilizing the SURPS' personality profiles to examine individual differences on substance use for each of the four profiles. However, it is also possible to investigate how constellations of traits within individuals are organized, using a person-oriented approach (Bergman and Magnusson 1997). The use of this approach might shed more light on how these constellations are associated with substance use in adolescents.

In sum, the present results suggest that in a large sample of early Dutch adolescents especially sensation seeking and hopelessness are strongly linked to the lifetime prevalence and age of onset of alcohol, tobacco, and cannabis use in early adolescents. Also, hopelessness and sensation seeking are found to be indicative of poly substance use. Building on these new insights, it will be crucial to conduct prospective analyses in the future to get more insight into how personality profiles can predict the development of substance use behaviors in adolescence and, vice versa, to determine whether substance use may affect adolescents' personality development. Further, recent studies investigated the effects of tailor-made interventions for the at-risk personality populations (Conrod et al. 2006, 2008, 2010). These studies show much promise for prevention efforts on excessive substance use, thus it seems that knowing who is at risk and what this risk is all about (i.e., only a risk for excessive use or also for early initiation) in combination with such effective prevention efforts might lead to an effective approach in diminishing (the negative effects of) substance use among (early) adolescents.

Acknowledgments This research was supported by a grant from The Dutch Ministry of Health, Welfare, and Sport.

Open Access This article is distributed under the terms of the Creative Commons Attribution Noncommercial License which permits any noncommercial use, distribution, and reproduction in any medium, provided the original author(s) and source are credited.

\section{Appendix}

See Table 3.

Table 3 Pearson correlations of personality profiles, substance use, age of onset, and poly substance use

\begin{tabular}{|c|c|c|c|c|c|c|c|c|c|c|}
\hline & 1 & 2 & 3 & 4 & 5 & 6 & 7 & 8 & 9 & 10 \\
\hline 1. Anxiety sensitivity & - & & & & & & & & & \\
\hline 2. Hopelessness & -.01 & - & & & & & & & & \\
\hline 3. Sensation seeking & .02 & $-.15 * *$ & - & & & & & & & \\
\hline 4. Impulsivity & $.24 * *$ & $.08 * *$ & $.37 * *$ & - & & & & & & \\
\hline 5. Lifetime alcohol & $.05 * *$ & $-.13 * *$ & $-.20 * *$ & $-.14 * *$ & - & & & & & \\
\hline 6. Lifetime tobacco & .01 & $-.19 * *$ & $-.18 * *$ & $-.18 * *$ & $.29 * *$ & - & & & & \\
\hline 7. Lifetime cannabis & -.01 & $-.06 * *$ & $-.10 * *$ & $-.10^{* *}$ & $.09 * *$ & $.23 * *$ & - & & & \\
\hline 8. Age of onset alcohol & .03 & $-.06 * *$ & $-.08 * *$ & $-.06^{*}$ & - & .04 & .02 & - & & \\
\hline 9. Age of onset tobacco & .03 & $-.08 *$ & .03 & .01 & .05 & - & .01 & $.38 * *$ & - & \\
\hline 10. Age of onset cannabis & .05 & $-.25 *$ & -.19 & -.01 & .17 & .05 & - & .23 & $.31 *$ & - \\
\hline 11. Poly substance use & $-.04 *$ & $.19 * *$ & $.24 * *$ & $.20 * *$ & $-.84 * *$ & $-.77 * *$ & $-.23 * *$ & -.04 & -.04 & -.12 \\
\hline
\end{tabular}

$* P<.05, * * P<.01$ 


\section{References}

Akse, J., Hale, W. W., III., Engels, R. C. M. E., Raaijmakers, Q. A. W., \& Meeus, W. H. J. (2004). Personality, perceived parental rejection and problem behavior in adolescence. Social Psychiatry and Psychiatric Epidemology, 39, 980-988.

Akse, J., Hale, W. W., III., Engels, R. C. M. E., Raaijmakers, Q. A. W., \& Meeus, W. H. J. (2007). Stability and change in personality type membership and anxiety in adolescence. Journal of Adolescence, 30, 813-834.

Andersen, A., Due, P., Holstein, B. E., \& Iversen, L. (2003). Tracking drinking behaviour from age 15-19 years. Addiction, 98, $1505-1511$.

Arnett, J. J. (1994). Sensation seeking. A new conceptualization and a new scale. Personality and Individual Differences, 16, 289-296.

Asparouhov, T., \& Muthén, B. (2009). Exploratory Structural Equation Modeling. Structural Equation Modeling, 16, 397-438.

Bailey, S. L., Flewelling, R. L., \& Rachal, J. V. (1992). The characterization of inconsistencies in self-reports of alcohol and marijuana use in a longitudinal study of adolescents. Journal of Studies on Alcohol, 53, 636-647.

Barrett, P. T., Petrides, K. V., Eysenck, S. B. G., \& Eysenck, H. J. (1998). The Eysenck Personality Questionnaire: An examination of the factorial similarity of $\mathrm{P}, \mathrm{E}, \mathrm{N}$, and $\mathrm{L}$ across 34 countries. Personality and Individual Differences, 25, 805-819.

Bentler, P. М. (1989). EQS structural equations program manual. Los Angeles: BMDP.

Bergman, L. R., \& Magnusson, D. (1997). A person-oriented approach in research on developmental psychopathology. Development and Psychopathology, 9, 291-319.

Brener, N. D., Billy, J. O., \& Grady, W. R. (2003). Assessment of factors affecting the validity of self-reported health-risk behavior among adolescents: Evidence from the scientific literature. Journal of Adolescent Health, 33, 436-457.

Breslau, N., Fenn, N., \& Peterson, E. L. (1993). Early smoking initiation and nicotine dependence in a cohort of young adults. Drug and Alcohol Dependence, 33, 129-137.

Brunelle, C., Assaad, J. M., Barrett, S. P., Vila, A. C., Conrod, P. J., Tremblay, R. E., et al. (2004). Heightened heart rate response to alcohol intoxication is associated with a reward-seeking personality profile. Alcoholism, Clinical and Experimental Research, $28,394-401$.

Carver, C. S., Johnson, S. L., \& Joormann, J. (2009). Two-mode models of self-regulation as a tool for conceptualizing effects of the serotonin system in normal behavior and diverse disorders. Current Directions in Psychological Science, 18, 195-199.

Caspi, A., Moffitt, T. E., Newman, D. L., \& Silva, P. A. (1996). Behavioral observations at age 3 years predict adult psychiatric disorders. Longitudinal evidence from a birth cohort. Archives of General Psychiatry, 53, 1033-1039.

Chen, C. Y., O'Brien, M. S., \& Anthony, J. C. (2005). Who becomes cannabis dependent soon after onset of use? Epidemiological evidence from the United States: 2000-2001. Drug and Alcohol Dependence, 79, 11-22.

Cloninger, C. R. (1998). A psychobiological model of temperament and character. In J. Richter, M. Eisemann, K. Bollow, \& D. Schläfke (Eds.), The development of psychiatry and its complexity. Munster (Germany): Waxmann Verlag GmbH.

Cloninger, C. R., Przybeck, T. R., \& Svrakic, D. M. (1991). The Tridimensional Personality Questionnaire: U. S. normative data. Psychological Reports, 69, 1047-1057.

Cohen, J. (1992). A power primer. Psychological Bulletin, 112, $155-159$.

Comeau, N., Stewart, S. H., \& Loba, P. (2001). The relations of trait anxiety, anxiety-sensitivity, and sensation seeking to adoles- cents' motivations for alcohol, cigarette, and marijuana use. Addictive Behaviors, 26, 1-24.

Conrod, P. J., Castellanos, N., \& Mackie, C. (2008). Personalitytargeted interventions delay the growth of adolescent drinking and binge drinking. Journal of Child Psychiatry, 49, 181-190.

Conrod, P. J., Castellanos-Ryan, N., \& Strang, J. (2010). Brief, personality-targeted coping skills interventions and survival as a non-drug user over a two-year period during adolescence. Archives of General Psychiatry, 67, 85-93.

Conrod, P. J., Pihl, R. O., \& Vassileva, J. (1998). Differential sensitivity to alcohol reinforcement in groups of men at risk for distinct alcoholic syndromes. Alcohol Clinical Experimental Research, 22, 585-597.

Conrod, P. J., Stewart, S. H., Comeau, N., \& Maclean, A. M. (2006). Efficacy of cognitive-behavioral interventions targeting personality risk factors for youth alcohol misuse. Journal of Clinical Child and Adolescents Psychology, 35, 550-563.

Cooper, M. L., Frone, M. R., Russell, M., \& Mudar, P. (1995). Drinking to regulate positive and negative emotions: A motivational model of alcohol use. Journal of Personality and Social Psychology, 69, 990-1005.

Costa, P. T., \& McCrae, R. R. (1992). Revised NEO personality inventory (NEO-PI-R) and NEO five-factor inventory (NEO-FFI) professional manual. Odessa, FL: Psychological Assessment Resources.

De Wit, D. J., Adlaf, E. M., Offord, D. R., \& Ogborne, A. C. (2000). Age at first alcohol use: A risk factor for the development of alcohol disorders. American Journal of Psychiatry, 157, 745-750.

Dishion, T. J., Capaldi, D. M., \& Yoerger, K. (1999). Middle childhood antecedents to progressions in male adolescent substance use: An ecological analysis of risk and protection. Journal of Adolescent Research, 14, 175-205.

Dolcini, M. M., Adler, N. E., \& Ginsberg, D. (1996). Factors influencing agreement between self-reports and biological measures of smoking among adolescents. Journal of research on adolescence, 6, 515-542.

Ellickson, P. L., Tucker, J. S., \& Klein, D. J. (2003). Ten-year prospective study of public health problems associated with early drinking. Pediatrics, 111, 949-955.

Engels, R. C. M. E., Knibbe, R. A., \& Drop, M. J. (1997). Inconsistencies in adolescents'self-reports of initiation of alcohol and tobacco use. Addictive Behaviors, 22, 613-623.

Eysenck, S. B., \& Eysenck, H. J. (1978). Impulsiveness and venturesomeness: Their position in a dimensional system of personality description. Psychological Reports, 43, 1247-1255.

Feigelman, W., Gorman, B. S., \& Lee, J. A. (1998). Binge drinkers, illicit drug users, and polydrug users: An epidemiological study of American collegians. Journal of Alcohol and Drug Education, $44,47-69$.

Flory, K., Lynam, D., Milich, R., Leukefield, C., \& Clayton, R. (2002). The relations among personality, symptoms on alcohol and marijuana abuse, and symptoms of comorbid psychopathology: Results from a community sample. Experimental and Clinical Psychopharmacology, 10, 425-434.

Galaif, E. R., \& Newcomb, M. D. (1999). Predictors of polydrug use among four ethnic groups: A 12-year longitudinal study. Addictive Behaviors, 24, 607-631.

Galizio, M., \& Stein, F. S. (1983). Sensation seeking and drug choice. International Journal of the Addictions, 18, 1039-1048.

Hale, W. W., III., Vander Valk, I., Akse, J., \& Meeus, W. (2008). The interplay of early adolescents' depressive symptoms, aggression and perceived parental rejection: A four-year community study. Journal of Youth and Adolescence, 37, 928-940.

Harakeh, Z., Scholte, R. H. J., De Vries, H., \& Engels, R. C. M. E. (2006). Association between personality and adolescent smoking. Addictive Behaviors, 31, 232-245. 
Hart, D., Atkins, R., \& Fegley, S. G. (2003). Personality and development in childhood: A person-centered approach. Monographs of the Society for Research in Child Development, 68, Serial No. 272.

Hibell, B., Guttormsson, U., Ahlström, S., Balakireva, O., Bjarnason, T., Kokkevi, A., et al. (2009). The 2007 ESPAD reportSubstance use among students in 35 European countries. Stockholm, Sweden: The Swedish Council for Information on Alcohol and Other Drugs (CAN).

Hu, L., \& Bentler, P. M. (1999). Cutoff criteria for fit indexes in covariance structure analysis: Conventional criteria versus new alternatives. Structural Equation Modeling, 6, 1-55.

Jackson, K. M., \& Sher, K. J. (2003). Alcohol use disorders and psychological distress: A prospective state-trait analysis. Journal of Abnormal Psychology, 112, 599-613.

Jaffee, J. W., \& D'Zurilla, T. J. (2009). Personality, problem solving, and adolescent substance use. Behavior Therapy, 40, 93-101.

Joiner, T. E. (2001). Negative attributional style, hopelessness, depression, and endogenous depression. Behaviour Research and Therapy, 39, 163-173.

Krank, M., Stewart, S. H., Wall, A. M., Woicik, P. B., \& Conrod, P. J. (submitted). Structural, concurrent, and predictive validity of the substance use risk personality scale in early adolescence.

Kremers, S. (2002). On your marks: Revising, testing and integrating stage models of smoking initiation. Doctoral dissertation, University of Maastricht.

Kremers, S. P., Mudde, A. N., \& De Vries, H. (2001). "Kicking the initiation": Do adolescent ex-smokers differ from other groups within the initiation continuum? Preventive Medicine, 33, 392-401.

Kuntsche, E., \& Jordan, M. D. (2006). Adolescent alcohol and cannabis use in relation to peer and school factors: Results of multilevel analyses. Drug and Alcohol Dependence, 84, 167-174.

Kuntsche, E., Overpeck, M., \& Dallago, L. (2008). Television viewing, computer use, and a hostile perception of classmates among adolescents from 34 countries. Swiss Journal of Psychology, 67, 97-106.

Kuntsche, E., Van der Vorst, H., \& Engels, R. C. M. E. (2009). The earlier the more? Differences in the links between age at first drink and adolescent alcohol use and related problems according to quality of parent-child relationships. Journal of Studies on Alcohol and Drugs, 70, 346-354.

Lacey, J. H., \& Evans, C. D. (1986). The impulsivist: A multiimpulsive personality disorder. British Journal of Addiction, 81, 641-649.

Loewenthal, K. M. (1996). An introduction to psychological tests and scales. London: UCL Press.

McCormick, R. A., Dowd, E. R., Quirt, S., \& Zegarra, J. H. (1998). The relationship of NEO-PI performance to coping styles, patterns of use, and triggers for use among substance abusers. Addictive Behaviors, 23, 497-507.

Monshouwer, K., Smit, F., De Graaf, R., Van Os, J., \& Vollebergh, W. (2005). First cannabis use: Does onset shift to younger ages? Findings from 1988 to 2003 from the Dutch national school survey on substance use. Addiction, 100, 963-970.

Monshouwer, K., Verdurmen, J., Van Dorsselaer, S., Smit, E., Gorter, A., \& Vollebergh, W. (2008). Jeugd en riskant gedrag 2007. Kerngegevens uit het peilstationsonderzoek scholieren. Utrecht, The Netherlands: Trimbos-insitute.
Muthén, L. K., \& Muthén, B. O. (1998-2007). Mplus user's guide (5th Ed.). Los Angeles, CA: Muthén \& Muthén.

O'Connor, R. C., Fraser, L., Whyte, M., MacHale, S., \& Masterton, G. (2008). A comparison of specific positive future expectancies and global hopelessness as predictors of suicidal ideation in a prospective study of repeat self-harmers. Journal of Affective Disorders, 110, 207-214.

Otten, R., Engels, R. C. M. E., \& Van den Eijnden, R. J. J. M. (2008). Smoking behavior in asthmatic and non-asthmatic adolescents: The role of smoking models and personality. Substance Use and Misuse, 43, 341-360.

Parra, G. R., O'Neill, S. E., \& Sher, K. J. (2003). Reliability of selfreported age of substance involvement onset. Psychology of Addictive Behaviors, 17, 211-218.

Peterson, R. A., \& Reiss, S. (1992). The anxiety sensitivity index manual (2nd ed.). Worthington, OH: International Diagnostic Systems.

Pulkkinen, L., \& Pitkänen, T. (1994). A prospective study of the precursors to problem drinking in young adulthood. Journal of Studies on Alcohol, 55, 578-587.

Reinherz, H. Z., Paradis, A. D., Giaconia, R. M., Stashwick, C. K., \& Fitzmaurice, G. (2003). Childhood and adolescent predictors of major depression in the transition to adulthood. American Journal of Psychiatry, 160, 2141-2147.

Reis, S. R., Peterson, R., Gursky, D., \& MacNally, R. (1986). Anxiety sensitivity, anxiety frequency and the prediction of fearfulness. Behaviour Research and Therapy, 24, 11-18.

Roberts, B. W., Walton, K. E., \& Viechtbauer, W. (2006). Personality traits change in adulthood: Reply to Costa and McCrae (2006). Psychological Bulletin, 132, 29-32.

Shall, M., Kemeny, A., \& Maltzman, I. (1992). Factors associated with alcohol use in university students. Journal of Studies on Alcohol, 53, 122-136.

Sher, K. J., Batholow, B. D., \& Wood, M. D. (2000). Personality and disinhibitory psychopathology: Alcoholism and anti-social personality disorder. Journal of Abnormal Psychology, 103, 92-102.

Smit, F., Monshouwer, K., \& Verdurmen, J. (2002). Polydrug use among secondary school students: Combinations, prevalences and risk profiles. Drugs: Education, Prevention and Policy, 9, $355-365$.

Spoont, M. (1992). Modulatory role of serotonin in neural information processing: Implications for human psychopathology. Psychological Bulletin, 112, 330-350.

Steiger, J. H. (1990). Structural model evaluation and modification. Multivariate Behavioral Research, 25, 173-180.

Stewart, S. H., Peterson, J. B., \& Pihl, R. O. (1995). Anxiety sensitivity and self-reported alcohol consumption rates in university women. Journal of Anxiety Disorders, 9, 283-292.

Tapert, S. F., Granholm, E., Leedy, N. G., \& Brown, S. A. (2002). Substance use and withdrawal: Neuropsychological functioning over 8 years in youth. Journal of the Neuropsychological Society, 8, 873-883.

Woicik, P. A., Stewart, S. H., Pihl, R. O., \& Conrod, P. J. (2009). The substance use risk profile scale: A scale measuring traits linked to reinforcement-specific substance use profiles. Addictive Behaviors, 34, 1042-1055.

Zuckerman, M. (1994). Behavioral expressions and biological bases of sensation seeking. New York: Cambridge University Press. 